\title{
UBER DIE ROLLE DES STICKSTOFFS IM FUTTERBAU
}

\author{
A. STÄHLIN ${ }^{1}$ ) \\ Justus Liebig-Universität, Giessen, Deutschland
}

Das Thema erscheint alt und überholt, aber nach den Fortschritten in der Erkenntnis der beiden letzten Jahrzehnte muss es sowohl für die Forschung als auch für die Praxis als wieder ganz aktuell angesehen werden. Es ist ja wohl immer angebracht, wenn alle 15-20 Jahre eine Bestandsaufnahme unserer Erkenntnisse über ein Wissensgebiet erfolgt, sofern überhaupt auf ihm geforscht und gearbeitet worden ist. Dann haben sich nämlich, wie jetzt in den Jahren seit 1945 auch auf dem Gebiet der Düngung und speziell der Anwendung von Stickstoff, soviele neue Erkenntnisse angesammelt, dass eine Inventur berechtigt ist, um das Erreichte und neu Erkannte zu überprüfen und neue Ziele der Forschung abzustecken.

So können jetzt beim Komplex Stickstoff viele Einzelgebiete neu überarbeitet werden, 1) die neu entwickelten und in Entwicklung befindlichen Stickstoff-Formen und ihre Zukunftsaussichten im Vergleich mit den alten Stickstoffdüngern, 2) die physiologischen Erkenntnisse von dem Einbau des Stickstoffs in die lebendige Substanz der Pflanze, die Änderungen der chemischen Zusammensetzung der Pflanze und die Auswertung dieser Erkenntnisse für den praktischen Pflanzenbau, speziell für die Düngung, auch in betriebswirtschaftlicher Hinsicht, 3) die Aufnahme der Stickstoffverbindungen aus der Pflanze sowohl in mineralischer als auch in organischer Form in den Tierkörper, ihre Aufspaltung bei der Verdauung und ihr Wiederauf- und Einbau in neue tierische Substanz, in Milch und Fleisch, Wolle und Haut, schliesslich ihre Wirkung auf die Gesundheit und die Lebensfunktionen des Tieres.

Bis in die 20er Jahre gab es an $N$-Formen $(1,4,8,9)$, neben dem alten Kalkstickstoff, nur Stickstoffdünger auf Salpetergrundlage oder als Ammoniakverbindungen (Darst. 1). Wenn auch die ersteren physiologisch-alkalisch und die letzteren physiologisch-sauer wirken, ist auf Dauergrünland die Stickstoff-Form wegen der Plastizität der Grasnarbe für eine längere Zahl von Jahren ziemlich gleichgültig. Nur bei jahrzehntelanger Anwendung derselben N-Form stellen sich, vor allem bei Schwefelsaurem Ammoniak, Mängel in der botanischen Zusammen-

1) Vortrag, gehalten am 20. 9. 63 vor der Universität Helsinki im Institut für Pflanzenbau in Viik. 
setzung heraus, die auf einer tiefgreifenden Versäuerung des Bodens beruhen. Der etwa hundertjährige Dauerversuch von Rothamsted auf reinen Mähflächen zeigt die daraus hervorgegangene Veränderung zu einem ausgesprochenen Ungräserbestand in erschreckender Deutlichkeit. Was hier zu wissenschaftlicher Erkenntnis vorexerziert worden ist, muss eine Mahnung zur Vermeidung jeglicher Einseitigkeit in der N-Düngung sein. Nach eigenen Versuchen sieht es so aus, als ob Salpeterdünger, wenn er Jahr für Jahr in grösseren Gaben verabreicht wird, ähnliche, wenn auch natürlich andere unerfreuliche Entwicklungen des Pflanzenbestandes auslösen würde. So nahm, zum Teil durch Ätzschaden an Untergräsern von' deren Konkurrenz befreit, Galium mollugo sehr zu.

Darstellung 1

Stickstoff-Formen als Düngemittel

Alt
Nitrat-Basis
Ammoniak-Basis
Cyanamid-Basis
Harnstoff-Basis

\author{
$\mathrm{Neu}$ \\ Urea-Basis \\ Crotonyl-Basis \\ Zusatz von Stoffen \\ mit hemmender Wirkung \\ auf die N-Löslichkeit
}

Nun ist der Verbrauch von Kalksalpeter und besonders von Kalkammonsalpeter in Deutschland sehr gestiegen im Gegensatz zum Schwefelsauren Ammoniak, das nur noch $10 \%$ des verwendeten Stickstoffdüngers ausmacht. Diese Entwicklung ist zu bedauern, weil die 3 essentiellen schwefelhaltigen Aminosäuren Cystin, Cystein und besonders Methionin im Eiweiss der Pflanzen in sehr viel geringeren Prozentsatz vertreten sind als in dem der Tiere. Ausserdem enthalten Schwefel das Vitamin $B_{1}=$ Aneurin, das trotz Neubildung im Pansen, wenn Schwefel vorhanden ist, günstig auf die Futterverwertung wirkt, wenn es im Futter enthalten war, ausserdem Vitamin H, das für den Plasma-Aufbau wichtig ist. Diese Stoffe können bei Schwefelmangel im Futter den Minimumfaktor für die Bildung von tierischem Eiweiss darstellen. In USA wird deshalb Methionin den Kraftfuttermitteln bereits zugesetzt.

Während allein bei der Düngung von Kreuzblütlern, Cruciferae, als Futterpflanzen wegen einer u.a. in Giessen festgestellten Erhöhung des Senfölgehaltes gegen eine starke Anwendung von Schwefelsaurem Ammoniak Bedenken erhoben werden müssen, sollten gerade bei den anderen Futterpflanzen, z.B. bei Futtergräsern und den von Natur stärker schwefelhaltigen Futterleguminosen, Versuche mit starker und stärkster Düngung von Schwefelsaurem Ammoniak zur Erhöhung des Gehaltes an den schwefelhaltigen Aminosäuren und Vitaminen gemacht werden. Natürlich wird diese Erhöhung nur in den Grenzen des arteigenen Eiweisses erfolgen können. Aber die Möglichkeit dazu ist bewiesen, weil ein gewisser Spielraum gegeben ist, ohne dass die Pflanzen Schaden leiden. Auf jeden Fall ist die Versorgung der Futterpflanzen mit Schwefel bei der Düngung mit Schwefelsaurem Ammoniak oder Superphosphat eine gern mitgenommene Nebenwirkung der 
N-Düngung, anstelle einer gezielten Düngung mit Schwefel. Auf jeden Fall wäre aus diesem Grund ein come back des Schwefelsauren Ammoniaks zu begrüssen.

$\mathrm{Zu}$ den alten Stickstoffdüngemitteln gehört auch Kalkstickstoff. Aber abgesehen von seiner unselektiven Ätzwirkung sind keine speziellen Erfahrungen mit ihm, seiner besonderen Wirkung und der möglichen Höhe seiner Gaben im Futterbau bekannt. Harnstoff aber spielt in Deutschland und speziell im Futterbau als Einzeldünger eine untergeordnete Rolle (4).

Aus Überlegungen arbeitswirtschaftlicher Einsparungen und pflanzenphysiologischer Ausnutzung müssen die leichte Wasserlöslichkeit und damit die schnelle Aufnehmbarkeit von fast allen alten Stickstoffdüngemitteln oft als Nachteil bezeichnet werden. Früher waren nur Wirtschaftsdünger und Kompost langsam und stetig fliessende Stickstoffquellen. Seit einigen Jahren sind indes langsam wirkende N-Düngemittel vorhanden, vor allem auf Urea- oder Crotonyl-Harnstoffbasis. Beim Ureaform handelt es sich um Harnstoff-Formaldehyd-Kondensate, von denen die einen Forscher in Deutschland eine entscheidende Bedeutung für die weitere Entwicklung der Düngertechnik erhoffen, bei deren Anwendung aber andere beim Vergleich mit Kalkammonsalpeter eine sehr viel geringere Ertragssteigerung und die Notwendigkeit der Anwendung von sehr viel grösseren Mengen an $\mathrm{N}$ zu gleichem Ertrag festgestellt haben $(1,8,9)$.

Versuche mit Ureaform zu Futterpflanzen sind in Deutschland anscheinend noch nicht angestellt worden. Aber bei Winterweizen betrug die Wirkung im Anwendungsjahr nur ca. $50 \%$, gegen $70-80 \%$ bei Kalksalpeter und Kalkammonsalpeter (1). Es war allerdings im folgenden Jahr eine deutliche Nachwirkung zu bemerken. Leider ist diese, wohl wegen einer grossen Abhängigkeit von der Jahreswitterung, nicht in allen Jahren der Fall gewesen, während eine Anreicherung an $\mathrm{N}$, wenigstens auf Ackerland im Winter vor allem wegen der Auswaschung, nicht möglich erscheint. Vielleicht ist es auf Dauergrünland mit seiner geschlossenen Narbe anders. Darauf deuten Ergebnisse hin, nach denen die Erträge mit UreaformDüngung besser über die Vegetation verteilt sind und das Wachstum im Spätsommer und Herbst nicht so rasch nachlässt. Andererseits ist eine gewisse Ertragshemmung konstatiert worden, die wohl auf eine schädigende Wirkung von Formaldehyd und seinen Oxydationsprodukten, vor allem der Ameisensäure, zurückzuführen ist.

Das Giessener Institut für Grünlandwirtschaft und Futterbau hat sich mit einer Reihe von Versuchen in die Erprobung des anderen neuartigen N-Düngers, des Floranids eingeschaltet, das aus Crototyl-di-harnstoff mit insgesamt $28 \% \mathrm{~N}$, davon $10 \%$ leicht löslich, besteht. Die Versuche umfassen solche mit FloranidStarkdüngung, im Vergleich mit Kalksalpeter, auf einer artenreichen Dauerwiese bei 3 und 6 Schnitten, weiter mit Floranid im Vergleich mit Kalkammonsalpeter zu den Futterpflanzenarten Festuca pratensis, Dactylis glomerata, Lolium perenne und Trifolium repens sowie zu 4 Sorten der Grasart Lolium perenne. Ausserdem läuft ein Versuch mit Floranidvolldünger und anderen Volldïngern auf Dauerwiese, darunter Nitrophoska Braun. Dieser neue Dünger ist mit einem Hemmstoff gegen eine rasche Lösung des Stickstoffs versehen.

Floranid schneidet in keinem unserer Versuche schlecht ab, ja es übertrifft 
Darstellung 2

Heuerträge von einer Dauerwiese unter dem Einfluss von Floranid

$(250 \mathrm{~kg} / \mathrm{ha} \mathrm{N})$

\begin{tabular}{|c|c|c|}
\hline \multicolumn{3}{|c|}{ (Relativwerte zu Kalksalpeter $=100)$} \\
\hline Jahr & 3-Schnitt & 6-Schnitt \\
\hline 1961 & .. 109 & 100 \\
\hline$\ldots \ldots \ldots \ldots \ldots \ldots \ldots \ldots \ldots \ldots$ & . 104 & 121 \\
\hline 1963 (bis 25. 7. 63, d.h. ohne & & \\
\hline 3. Schnitt) $\ldots \ldots \ldots \ldots \ldots \ldots \ldots$ & 97 & 112 \\
\hline
\end{tabular}

den Kalksalpeter stets (Darst. 2). Immerhin kommt auch bei diesen Versuchen klar heraus, dass die Wirkung je nach der Witterung ganz verschieden gross sein kann. Ebenso wird die Hoffnung auf eine Nachwirkung oft enttäuscht. Die Lösung des Stickstoffs im Floranid erfolgt anscheinend in Parallele mit der Intensität des Bodenlebens sehr langsam, sowohl bei kühler und kalter Frühjahrswitterung als auch bei Sommerdürre. Im ersten Schnitt war Floranid immer überlegen, wohl wegen der Wirkung der in ihm enthaltenen leicht löslichen N-Verbindungen. Danach aber war in der übrigen Vegetationszeit deutlich zu sehen, dass der im Floranid enthaltene Stickstoff schwer löslich ist; denn die Floranidparzellen waren den ganzen Sommer über heller grün und weniger wüchsig. Sie zeigten nur am Ende der Vegetationszeit, als der Stickstoff des Kalkammonsalpeters weitgehend oder ganz verbraucht war, eine dunklergrüne Färbung als dessen Teilstücke. Die Leistung des Floranids liegt, wenn man Kalksalpeter $=100$ setzt, in den bisherigen Versuchsjahren stets höher als bei Kalksalpeter. Wahrscheinlich wäre sie noch höher gewesen, wenn nicht das Bodenleben bei jedem Schnitt infolge der Austrocknung der obersten Bodenschichten gestört worden wäre. Auch soll der von den höheren Pflanzen nicht aufgenommene Floranid-Stickstoff, sobald er gelöst ist, von Mikroorganismen des Bodens in ihre Körpersubstanz eingebaut werden, und zwar so fest, dass die Freigabe an die höheren Pflanzen nicht geschieht.

Nach diesen noch nicht abgeschlossenen Versuchen sieht es so aus, als ob Floranid besser abschneiden könne als Ureaform. Im Gegensatz zu der Gefahr des Ausbrennens der Narbe mit Salpeterdünger ist eine Verätzung auch bei sehr hohen Gaben von Floranid mit $750 \mathrm{~kg} / \mathrm{ha} \mathrm{N}$ in einer Gabe nicht zu befürchten. Die Aussichten für Floranid sind also im ganzjährigen Futterbau als ausgesprochen günstig zu beurteilen, auch für den aus ihm hergestellten Volldünger, wegen der schonenden Wirkung auf die Kleepflanzen und ihren Ertragsanteil. So ist die Entwicklung der langsam wirkenden Stickstoffdünger ganz allgemein mit Wohlwollen abzuwarten, da auf diesem Gebiet weiter von den Firmen gearbeitet wird.

Die kleeverdrängende Wirkung von Stickstoff auf Dauergrünland und in Kleegrasbeständen ist seit langem bekannt. Um zu prüfen, ob man sie, z.B. durch Aussetzen der N-Düngung, vermeiden könne, laufen am Giessener Insitut für Grünlandwirtschaft und Futterbau 2 Versuche nach gleichem Plan. In der Darstellung 
3 sind vor allem die Daten über die Erhaltung des wertvollen Kleeanteils bei NWechseldüngung mit denen von Volldüngung und Kaliphosphat zu vergleichen. Es scheint nach 6jähriger Versuchsdauer der Schluss berechtigt, dass ein erfreulicher Prozentsatz von Grünlandleguminosen lediglich durch den jährlich erfolgenden Wechsel der Stickstoffgabe vor und nach dem ersten Schnitt erhalten bleiben könne. Freilich kommt der Bestand infolge der Wechseldüngung nie zur Konsolidierung.

Darste 11 u $\mathrm{n} \quad 3$

Botanische Zusammensetzung einer Dauerwiese unter dem Einfluss von Stickstoffdüngung

(Beispiel 1. Schnitt 1960-63)

\begin{tabular}{|c|c|c|c|c|}
\hline & 0 & PK *) & NPK **) & N-Wechseldüngung ${ }^{* * *}$ ) \\
\hline$\ldots \ldots \ldots \ldots$ & 56 & 62 & 80 & 66 \\
\hline Leguminosen- $\% \quad \ldots \ldots$ & 19 & 22 & 10 & 17 \\
\hline Kräuter- $\% \ldots \ldots \ldots \ldots$ & 25 & 16 & 10 & 17 \\
\hline
\end{tabular}

*) $90 \mathrm{~kg} / \mathrm{ha} \mathrm{P}_{2} \mathrm{O}_{5}$

$120, \mathrm{~K}_{2} \mathrm{O}$
**) $60 \mathrm{~kg} / \mathrm{ha} \mathrm{N}$

$90 \div \mathrm{P}_{2} \mathrm{O}_{5}$

$120, \mathrm{~K}_{2} \mathrm{O}$
***) 196

1961: 60 N zum 1. Schnitt

1962: $60, \mathrm{~N}$ zum 2. Schnitt

1963: 60 N zum 1. Schnitt

Darstellung 4

Stickstoffmenge je ha und Ertrag

\begin{tabular}{|c|c|c|c|c|}
\hline & \multicolumn{2}{|c|}{$\mathrm{kg} / \mathrm{ha} \mathrm{N}$} & \multicolumn{2}{|c|}{ Ertrag in $\mathrm{d} z /$ ha $\mathrm{Heu}$} \\
\hline & früher & jetzt & früher & jetzt \\
\hline \multicolumn{5}{|l|}{ Ackerfutterbau: } \\
\hline Kleearten $\quad \ldots \ldots \ldots \ldots \ldots$ & $0-20$ & $0-20$ & $40-100$ & $40-100$ \\
\hline$\ldots \ldots \ldots \ldots \ldots$ & $0-60$ & $0-60$ & $50-120$ & $50-120$ \\
\hline Nichtleguminosen..$\ldots \ldots$ & $50-100$ & $100-200$ & $80-120$ & $100-180$ \\
\hline \multicolumn{5}{|l|}{ Dauer- $u$. Wechselgrünland: } \\
\hline Wiesen $\quad \ldots \ldots \ldots \ldots \ldots \ldots$ & $0-50$ & $40-200$ & $20-80$ & $50-200$ \\
\hline Weiden $\ldots \ldots \ldots \ldots \ldots$ & $60-100$ & $100-300$ & $1500-3000$ & $4000-8000$ \\
\hline
\end{tabular}

Es ist bereits im ersten Kapitel über neuartige $\mathrm{N}$-formen auch immer etwas über die Höhe der Stickstoffdüngung $(2,3,5,7,10,11,13,14,15)$ gesagt worden. Trotzdem ist noch eine kurze Angabe der in Mittel- und Westeuropa z.Zt. angewendeten Mengen von Stickstoffdüngemitteln notwendig. Noch zu Beginn der 30er Jahre war eine hohe Gabe für alle landwirtschaftlichen Kulturen, schon wegen des hohen Preises für die N-Einheit, nicht üblich (Darst. 4). Aber in den letzten 
Jahren kletterte die verabreichte Stickstoffmenge dauernd nach oben. Diese Feststellung trifft nicht nur für Getreide, Zuckerrüben und andere Marktfrüchte zu, sondern auch für Feldfutterpflanzen und für Grünland. Der Verbrauch an N im Futterbau ist unter dem Einfluss von staatlicher Beratung und der Stickstoffindustrie auch deshalb gestiegen, weil im Hauptfruchtfutterbau an die Stelle des teueren und in ausländischen Herkünften unsicheren Rotklees (Trifolium pratense) und der Luzerne (Medicago sativa) sehr oft Kleegras, reines Gras oder Silomais getreten ist $(13,14)$. Im Zwischenfruchtbau aber trat ein Wechsel von dem teueren Leguminosengemenge zu Nicht-Leguminosen wie Grünfuttermais und Grünhafer, diese beiden auch statt Futterrüben, sowie von Kreuzblütlern und Süssgräsern ein. Alle diese Arten und Artengruppen sind N-dankbar.

Jetzt erhalten Futterroggen und Futterraps, dieser sowohl in der Winter- als auch in der Sommerform, Futterhafer und alle anderen Nicht-Hülsenfrüchtler unter den Futterpflanzen Stickstoffmengen, die in gut geleiteten Betrieben weit über $100 \mathrm{~kg} /$ ha $\mathrm{N}$ hinausgehen. Das gleiche gilt für das Wechselgrünland, d.h. die Leys, und für die alten Wiesen und Weiden.

Noch vor wenigen Jahren war in beiden Teilen Deutschlands Stickstoff auf den Mähflächen verpönt und man wollte die Steigerung des Ertrages allein mit starken Kaliphosphatgaben, d.h. durch Förderung der natürlichen Stickstoffquelle aus den Knöllchenbakterien der Grünlandleguminosen, erreichen. Aber jetzt weiss man, dass Höchsterträge ohne N-Gaben nicht möglich sind, so wichtig auch eine Grunddüngung mit Kaliphosphat als Grundlage für eine befriedigende Menge und Qualität des Futters ist. Es ist allerdings nach Beobachtungen und Versuchen anzunehmen, dass auf den verschiedenartigen Pflanzenbeständen der mitteleuropäischen Wiesen nicht jedes Jahr die Stickstoffpeitsche wegen ihrer egalisierenden Wirkung auf die botanische Zusammensetzung der Bestände angewendet werden sollte. So eignen sich grasreiche Wiesen, wie sie vorwiegend in Norddeutschland vorkommen, bedeutend besser für eine rentable N-Düngung als die in Süddeutschland weit verbreiteten klee- und kräuterreichen Mähflächen. Wahrscheinlich wäre es richtig, wenn Stickstoffsteigerungsversuche bis 300 und $400 \mathrm{~kg} / \mathrm{ha} \mathrm{N}$ auf möglichst vielen Standorten vorgenommen würden, damit auch die finanziell optimale Höhe der Stickstoffgabe auf Mähflächen bekannt würde; denn darüber sind sich die Gelehrten in Deutschland noch nicht einig geworden.

Auf den Dauerweiden wirkt eine N-Steigerung sehr viel gleichmässiger in Richtung auf einen hohen Mengenertrag, weil der Pflanzenbestand einerseits fast über alle Standortsunterschiede hinweg durch eine intensive Düngung und Nutzung zu einer rein anthropogenen Zusammensetzung egalisiert wird, andererseits weil auf einer Intensivweide sich nur diejenigen Arten halten können, die einseitig hohe N-Gaben lohnen. Aus diesem Grunde haben einzelne Landwirte in den Niederlanden Mengen von mehr als $400 \mathrm{~kg} / \mathrm{ha} \mathrm{N}$ auf den Weiden ihrer Betriebe mit Erfolg angewendet. Dadurch wurde nicht nur die Bodenfruchtbarkeit, sondern auch die Menge des Aufwuchses und die Leistung der Flächen sehr verbessert. Mit dieser hohen N-Gabe aber scheint auch in den klimabegünstigten Niederlanden eine dreifache Grenze erreicht zu sein: 1) auf dem Gebiet der Pflanzensoziologie, weil sich 
die Weidenarbe ganz einseitig zu einem fast reinen Bestand von Lolium perenne, d.h. zu einem Gesellschaftsfragment im pflanzensoziologischen Sinne, entwickelt hat; 2) auf dem Gebiet der Pflanzen- und Tierphysiologie, weil die Assimilationsleistung von Lolium perenne zeitweise infolge der Ungunst kühler und sonnenarmer Witterung nicht mehr zur Verarbeitung des aufgenommenen Stickstoffs in vollwertiges Pflanzeneiweiss genügt hat, dieser als Nitrat oder Amide im Zellsaft geblieben ist und bei der Aufnahme solch jungen und jüngsten Futters von den Tieren schlechter ausgenutzt worden ist, bis der tierische Organismus zuletzt krank geworden ist; 3) auf dem Gebiet der Rentabilität, weil die einzelne Stickstoffeinheit infolge des Gesetzes vom abnehmenden Bodenertrag einen immer geringer werdenden Ertragszuwachs erzeugt hat, ganz abgesehen davon, dass durch die schlechtere Ausnutzung der stickstoffhaltigen Substanz durch die Tiere die Rentabilität einer hohen N-Gabe gedrückt worden ist (2).

Ausserdem können anscheinend nicht alle Stoffe, die zu einem raschen Austreiben der Pflanzen nach jeder Nutzung und im Frühjahr notwendig sind, durch N-Düngung voll ersetzt werden. In Versuchen mit ganz anderer Fragestellung, nämlich zur Klärung der chemischen Identität von Reservestoffen und Gräsern und zur Klärung der Frage, ob ein Ersatz der bei Herbstnutzung von Grassamenbeständen verbrauchten Reservestoffe durch N-Düngung möglich sei, war zwar bei der Samenernte von Gräserarten kein Unterschied im Ertrag festzustellen; wohl aber in der Wuchsfreudigkeit im Frühjahr und nach dem Samenschnitt. D.h. es war eine negative Nachwirkung der Herbstnutzung vorhanden, über die Samenernte hinweg und trotz einer zusätzlichen $\mathrm{N}$-Gabe von $40 \mathrm{~kg} / \mathrm{ha}$, die als Ausgleich für die Herbstnutzung gedacht war.

Um bei Weidenutzung in dem durch N-Düngung rascher werdenden Umtrieb den ganzen Aufwuchs durch die Weidetiere oder durch Einsäuerung zu nutzen, muss die Besatzstärke mit Rindvieh vergrössert werden. Darunter leidet die Leistung des Einzeltieres, obwohl die der einzelnen Koppel noch steigt. Deswegen bestossen holländische Zuchtbetriebe, bei denen es um die letzte Ausnutzung der tierischen Leistungsanlage zu höchsten Milchleistungen geht, ihre Weiden mit einem Bruchteil der Milch- und Mastbetriebe und düngen dementsprechend gering, nur mit $60-100 \mathrm{~kg} / \mathrm{ha} \mathrm{N}$, damit die Tiere bei einer für ihre Formgestaltung notwendigen Bewegung das aufwachsende Futter bewältigen können.

Hier sehen wir also schon pflanzen- und tierphysiologische Grenzen, die vielleicht durch unsere Kunst noch etwas hinausgeschoben werden können, damit die Rentabilitätsgrenze von gesteigerten Stickstoffgaben später erreicht wird. Aber es dürfte schwer fallen, die durch klimatische Ungunst in den deutschen Mittelgebirgen und in Finnland gesetzten Grenzen ganz zu kompensieren, weil die Vegetationszeit und damit die Zeit zur Assimilation und Eiweissynthese immer kürzer werden.

Es können zwar anscheinend die Weiden im Gegensatz zu reinen Mähflächen von einem tüchtigen Weidewirt zu sehr hohen Leistungen getrieben werden. Das beweist z.B. der beste Weidebetrieb der Bundesrepublik, der nicht, wie man meinen sollte, in einer für Grünlandnutzung günstigen feucht-warmen Lage mit langer Vegetationszeit, z.B. am Niederrhein in dem an die Niederlande grenzenden Teil 
von Westdeutschland, liegt, sondern in dem rauhen und bodenmässig ungünstigen Fichtelgebirge.

Wenn man aber das gerade durch Stickstoffdüngung stark beeinflussbare Leistungspotential der mitteleuropäischen Weiden berechnen will und die dementsprechenden Angaben in den letzten 10 Jahren verfolgt, dann sieht man, wie das Leistungspotential von ca. 5000 auf 8000 Stärkeeinheiten in die Höhe geklettert ist. Mit anderen Worten, die $10 \%$ besten Betriebe, deren Ertrag das Potential bedeutet, haben in den letzten Jahren die Leistung ihrer Weiden ständig steigern können. Das geschah vielleicht gar nicht mehr durch eine Erhöhung der pflanzlichen Wuchsleistung, sondern lediglich durch eine Änderung der Weideführung zu besserer Ausnutzung des Aufwuchses und zu einer Verringerung der bei Nichtausnutzung eintretenden Nährstoffverluste. Es stecken ja in jedem Weidetagebuch sehr viele kaum fassbare Imponderabilien, die bis zur gelungenen oder verfehlten Wetterprophetie des Weidewirtes reichen, ob er eine Koppel noch abmähen darf oder als Futterreserve stehen lassen muss.

Eine reichliche N-Gabe ist immer eine Sicherung gegen ein Auftreten von Futtermangel; denn gut ernährte Futterbestände überstehen Trockenperioden, wie sie jedes Jahr in Mitteleuropa vorkommen, bedeutend besser als magere Bestände. Das wird fast jedes Jahr von Betrieb zu Betrieb in Mitteleuropa demonstriert. Vor allem ist die Weidenarbe so dicht, dass trotz der deutlichen Verkürzung der Wurzeln infolge intensiver Nutzung wenig Nährstoffe durch Auswaschung verlorengehen. Je stärker aber mit $\mathrm{N}$ gearbeitet wird, desto mehr wird diese Erscheinung einer seichten Narbe ausgeglichen, bis sogar die Bodenfruchtbarkeit durch Vertiefung und bessere Zersetzung der organischen Schicht infolge regeren Bodenlebens, vor allem infolge der Vermehrung der Zahl von Regenwürmern, erhöht wird.

Wenn wir bei der Feststellung des Rohproteingehaltes mit dem Produkt $\mathrm{N} \% \times$ Faktor 6,25 rechnen, ist dies nur ein grober Durchschnitts- und Annäherungswert, weil die stickstoffhaltige Substanz $(6,12,16)$ in der grossen Mannigfaltigkeit der Eiweissstoffe, ihrer Vorstufen, Bausteine und Abbaustufen um mindestens einen Gehalt von 15-18 \% N schwankt. Zum Rohprotein gehören nicht nur echte Eiweissstoffe und ihre Bausteine in Form von Aminosäuren, sondern auch Vorstufen wie Amide und Zerfallsprodukte bis Ammoniak, ferner die ebenfalls stickstoffhaltigen Alkaloide, deren Bedeutung und Funktion für den pflanzlichen Organismus noch unklar ist, deren grossenteils erwiesene Giftigkeit aber sie im negativen Sinn für die Tierernährung und den Futterbau interessant machen.

Durch N-Düngung wird im allgemeinen der Rohproteingehalt stark angehoben (Darst. 5). Nur bei niedrigen Gaben bis etwa $30 \mathrm{~kg} / \mathrm{ha} \mathrm{N}$ erfolgt eine so starke Vermehrung der Grünmasse, dass der Rohproteingehalt meist sogar niedriger als bei Ungedüngt ist. Aber das sind Ausnahmen von der Regel, nach der Pflanzen, die reichlich mit $\mathrm{N}$ versorgt werden, in dem gleichen phänologischen Entwicklungsstadium bis 25 und mehr \% Rohprotein gegenüber $8-10 \%$ bei keiner N-Düngung aufweisen. Neben der Menge ist indes die Zusammensetzung der N-haltigen Substanz wichtig. Wohl können Wiederkäuer Eiweissvorstufen und Eiweissabbauprodukte, sogar mineralische Stickstoffverbindungen ausnutzen, jedenfalls mehr als Tiere mit einhöhligem Magen. Aber auch bei Wiederkäuern spielen Aufbau 
Veränderung der Zusammensetzung von Futterpflanzen durch starke Stickstoffdüngung

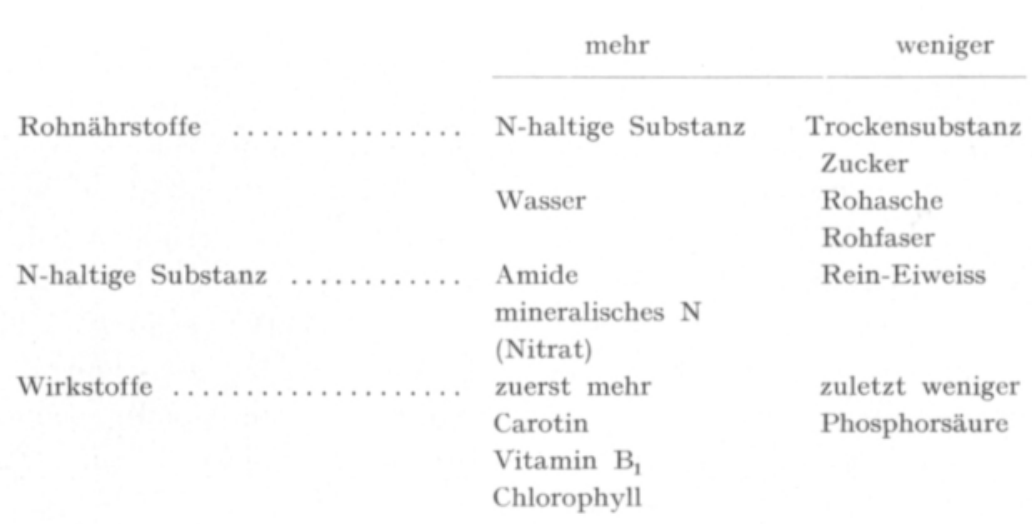

und Zusammensetzung der N-haltigen Substanz eine ganz grosse Rolle. Durch die N-Düngung werden nun die Bildung der einzelnen Aminosäuren und damit ihr Anteil im Rohprotein ganz verschieden beeinflusst, so dass ein stark mit N versorgter Aufwuchs womöglich nicht mehr vollwertig ist. Da gleichzeitig mit der Zusammensetzung des Rohproteins auch der Gehalt an Chlorophyll und Xanthophyll sowie an den meisten Vitaminen beeinflusst wird, kann der Wert eines solchen mastig gewachsenen Futters stark und meist zum Schlechteren hin verändert werden; das ergeben u.a. neueste eigene Untersuchungen bezüglich des Chlorophyllund Carotingehaltes.

Auch der Gehalt an Rohasche wird gesenkt, während der an Rohfaser bei gesteigerten $\mathrm{N}$-Gaben zuerst ansteigt und erst von einer gewissen $\mathrm{N}$-Menge an etwa konstant bleibt. In dem Rohfaser genannten Komplex von Stoffen treten ebenfalls Änderungen ein. Es ist ausdrücklich darauf hinzuweisen, dass in der jetzigen Zeit mit ihren von der tierischen Leistung her gesteigerten Anforderungen an die pflanzliche Qualität die Rohfaserwerte nicht mehr genug über den Futterwert aussagen.

Die stets konstatierte Senkung des Trockensubstanzgehaltes ist eine unangenehme Begleiterscheinung bei jeder stärkeren N-Düngung, sowohl für Heuwerbung als auch beim Einsäuern. Vor allem aber wird die chemische Zusammensetzung der N-haltigen Substanz in den Futterpflanzen grundlegend verändert, indem bei Gräsern und Grünhafer die Differenz von Gesamtstickstoff und Eiweissstickstoff um das Doppelte, von 20 auf $40 \%$ des Gesamtstickstoffs, zunimmt $(6,16)$. Parallel dazu steigt auch der Gehalt an Amid-Stickstoff. In stark mit N getriebenem Futter scheint auch der Gehalt an potentiellen Aminosäuren sehr viel geringer zu sein. Ausserdem kann es auch im gemässigten Klima von Mittel- und Westeuropa vorkommen, nicht nur bei jähen Wetterstürzen mit trübem Wetter in den Tropen und Subtropen, dass Stickstoff in mineralischer Form, und zwar meist als Nitrat befunden wird. Dann war es zu einem Abbruch oder krassem Rückgang der Assimilation, nach reicher Stickstoffaufnahme bei sogenanntem Wachswetter, gekom- 
D a rste 11 u ng 6

Wirkung von starker Stickstoffdïngung auf Pflanzenbestand und Tier

$$
\text { Positiv }
$$

mehr junges, weiches Futter $=$ Zunahme der Flächenleistung

mehr N-haltige Substanz und Carotin = höherer Futterwert grössere Aufnahmewilligkeit $=$ Zunahme der tierischen Leistung

Zunahme der tierischen Leistung

Negativ

in Mischbeständen, besonders auf Dauergrünland Egalisierung der botanischen Zusammensetzung, mehr Gräser, weniger Geschmackstoffe = geringerer Futterwert

sehr viel $\mathrm{N}$-haltige Substanz nicht eiweissartiger Natur, weniger Aschenbestandteile, wenig Ballaststoffe $=$ geringerer Futterwert

Disharmonie der Nährstoffzusammensetzung

Dysharmonie der Nährstoffzusammensetzung
Abnahme der Aufnahmewilligkeit $=$ Abnahme der tierischen Leistung

(bei noch steigender Flächenleistung)

schlechtere Ausnutzung des Futters

$=$ Abnahme der tierischen Leistung,

Mehrkosten durch Ergänzungsfütterung

Störung des Stoffwechsels $=$ starke Abnahme der tierischen Leistung

Durchfall, Aufblähen, Tetanie

$=$ Zusammenbruch der tierischen Leistung

men. So sind Werte festgestellt worden, mit denen der Schwellenwert für die Schädlichkeit bei Tieren überschritten worden war. Dann werden die Tiere krank, sie bekommen Durchfall, blähen auf und können sterben. Auf jeden Fall entscheidet über den Wert der Futterpflanzen sehr stark die Zusammensetzung des pflanzlichen Eiweisses (Darst. 6), und zwar der Anteil der zur Bildung von tierischem Eiweiss essentiellen Aminosäuren.

\section{$Z u s a m m$ enfassung}

Von den Stickstoff-Formen, die seit langem als N-Düngemittel gebräuchlich sind, hat jede ihre Vorteile und Nachteile für die N-Versorgung von Futterpflanzen. Alle zusammen werden sehr rasch, z.T. nach Umwandlung in Salpeter, von den Pflanzen aufgenommen und deshalb ist ihre Wirkung meistens sehr schnell im ersten Aufwuchs verpufft. Aber die beiden jetzt in Deutschland in Prüfung befindlichen langsam löslichen N-Düngemittel, der Ureaform und das Floranid, weisen in der grossen Abhängigkeit ihrer Löslichkeit von der derzeitigen Witterung oft eine schlechtere Wirkung als die althergebrachten Düngemittel auf. Ihr Preis, der jetzt für landwirtschaftliche Nutzung vollkommen undiskutabel ist, dürfte in Angebot und Nachfrage eine starke Senkung erfahren können.

Die Ansichten über die Höhe der Stickstoffdüngung sind bei Futterpflanzen seit dem Zweiten Weltkrieg in Fluss geraten. Während wir auf der Intensivweide relativ klar sehen, nämlich dass wir sehr hoch, in günstiger Klimalage bis $300 \mathrm{~kg} / \mathrm{ha}$ 
$\mathrm{N}$, düngen dürfen, kann die Menge, die von den verschiedenen Pflanzengesellschaften der Dauerwiese ausgenutzt wird, noch nicht sicher mit festen Ratschlägen angegeben werden. Im Ackerfutterbau treten, wie auf dem Dauergrünland, nach stärkeren $\mathrm{N}$-Gaben Veränderungen in der stofflichen Zusammensetzung des Aufwuchses ein, die tierphysiologische Bedeutung haben, wenn nicht eine Kompensation mit anderen Futtermitteln vorgenommen wird, die vor allem eine Ergänzung in Form von essentiellen Aminosäuren besitzen.

Es zeichnen sich noch andere natürliche Grenzen ab, z.B. nach starker Nutzung in der unvollkommenen Ersetzung von Reservestoffen in der Graspflanze durch Stickstoffdüngung, sowie in der unvollständigen Verarbeitung des in mineralischer Form aufgenommenen Stickstoffs durch die Pflanze. Wahrscheinlich dürfte indes in den meisten landwirtschaftlichen Betrieben die Höhe der verabreichten Stickstoffgaben letztlich mehr von betriebswirtschaftlichen Erwägungen als von solchen pflanzen- oder tierphysiologischer Art bestimmt werden.

\section{LITERATURNACHWEIS}

(1) Atanasıu, N. 1955/56. Studien über Ertragswirkung von schwerlöslichen Stickstoff-Düngemitteln. Landw. Forsch. So.-H. 7: 108-113.

(2) BOER, P. B. DE 1962. Meine Erfahrungen mit intensiver Grünlandbewirtschaftung. Vortrag Justusvon Liebig-Preis-Stiftung zu Hamburg, Kiel.

(3) Brandsch, F. 1956. Ertragssteigerung im Futterbau. DLG, Frankfurt/M.

(4) Buchner, A. \& Kradel, J. 1961/62. Die Anwendung von Harnstoff als Düngemittel. Z. Ackeru. Pflanzenbau 114: 1-22.

(5) Bürger, K. \& Beuster, K.-H. \& Herforth, G. \& Terkamp, E. 1961. Unsere Gräser im Futterund Samenbau. Boden u. Pflanze Nr. 9, Ruhrstickstoff A.G., Bochum.

(6) Burghardt, H. 1960. Eiweisszusammensetzung und Futterqualität. Mitt. DLG 75: $1423-1424$.

(7) Grafenschäfer, E. 1953. Beitrag zur Kenntnis des Anbaues von Welschem Weidelgras am Niederrhein. Z. Acker- u. Pflanzenbau 96: 477-492.

(8) JUNG, J. 1961. Uber langsam wirkende Stickstoffverbindungen, insbesondere Crotonylidendiharnstoff. Z. Pflanzenern., Düng., Bodenk. 94: 39-47.

(9) Kaltofen, H. 1963. Zur Frage der Anwendung von Ureaform unter besonderer Berücksichtigung des Grünlandes. Z. Landeskultur 4: 91-102.

(10) KögeL, W. 1961/62. Uber Ursachen des Rückgangs des Rotkleeanbaues. Z. Acker- u. Pflanzenbau 114: $32-52$.

(11) Köhnleın, J. \& Wıтzıg, J. 1961. Ergebnisse mehrjähriger Anbauversuche mit einjährigen Futterpflanzen. Kieler Milchwirtschaftl. Forschungsber. 13: 315-360.

(12) Mengel, K. 1961. Ernährung und Stoffwechsel der Pflanze. VEB Gust. Fischer, Jena.

(13) SAchs, E. 1956. Kleegrasmischungen und Düngung. Bayer. Landw. Jb. 33: 319-333.

(14) 1961. Luzernegrasgemische und Düngung. Ibid. 33: 431-445.

(15) SchulzE, E. 1961. Anwendung und Wirkung der Stickstoffdünger bei Feldfrüchten und Dauergrünland. Der Stickstoff. Fachverb. Stickstoffind. Düsseldorf.

(16) Wiesemóleer, W. 1963. Stickstoffdüngung und Inhaltsstoffe in verschiedenen Futterpflanzen. Z. Landw. Vers.-Untersuchungswesen 9: 199-213. 
S E L O T U S:

TYPEN MERKITYKSEST A REHUNVILJELYSSÅ

\section{A. Stählin}

Justus Liebig-Yliopisto, Giessen, Saksa

Toisen maailmansodan jälkeen on erityisesti selvitelty seuraavia typpilannoituksen käyttöőn rehunviljelyssä liittyviä kysymyksiä:

1) Uudet vastakehitetyt typpilannoitteet verrattuina aikaisemmin käytettyihin.

2) Voimakkaan typpilannoituksen aiheuttamat muutokset kasvin valkuaiskoostumuksessa.

3) Typpiyhdisteiden eläinruumiissa tapahtuva muuttuminen eläinvalkuaiseksi ja sen vaikutus eläinten terveyteen ja tuotantoon.

Entisissä typpilannoitteissa on typpi joko nitraatti- tai ammoniumtyppenä. Kokeet ovat osoittaneet, ettă lukuunottamatta tapauksia, jolloin lannoitus on erittäin yksipuolinen tai poikkeuksellisen voimakas, on yhdentekevää, kummassa muodossa typpi rehukasveille annetaan. Yhteistä kaikille vanhoille typpilannoitteille on, että niiden sisältämä typpi tulee käytetyksi kasvien hyväksi sangen nopeasti ja lyhyessä ajassa. Tämän haitan poistamiseksi on kehitetty uusia, hitaasti vaikuttavia ureaja krotonylaldehydiurea-yhdisteisiin (Floranid) perustuvia lannoitteita. Voidaan myös lisätä lannoitteisiin aineita, jotka hidastavat typen liukenemista. Uusien typpilannoitteiden vaikutusteho on kuitenkin suuressa määrin riippuvainen käytön jälkeisen ajan sääoloista.

Typpilannoitteiden käyttömääriä on Saksassa sodan jälkeen voimakkaasti lisätty varsinkin palkokasveihin kuulumattomien rehukasvien sekä laitumien osalta. Voimaperäisessä laidunviljelyssä voidaan käyttää jopa $300 \mathrm{~kg} / \mathrm{ha} \mathrm{N}$. Pitkäikäisten nurmien ja peltoviljelyssä tuotettujen rehukasvien sadon koostumuksessa todetaan kuitenkin voimakkaan typpilannoituksen seurauksena muutoksia, jotka puolestaan aikaansaavat haitallisia fysiologisia vaikutuksia kotieläimissä. Sadon raakaproteiinin laatu huononee, ei vain siksi, että palkokasvien osuus sadosta pienenee, vaan myös siitä syystä, että kasvien ottama typpimäärä ei kokonaan muunnu puhdasvalkuaiseksi, vaan osa siită voi jäädä nitraattimuodossa kasvien solunesteeseen. Vaikka märehtijät pystyvätkin käyttämäăn hyväkseen valkuaisaineiden esiasteita, on pelättävissä, että ylisuurien typpimäärien käyttö rehunviljelyssä aiheuttaa karjalle vakaviakin terveydellisiä haittoja. Useimmiten kuitenkin liiketaloudelliset tekijät määräävät käytettävän typpilannoituksen voimakkuuden kussakin tapauksessa jo ennen kuin kasvi- ja eläinfysiologiset haittavaikutukset käyvät ilmeisiksi. 